\title{
Design Methodology Framework for Cyber-Physical Products
}

\author{
Haider AL-Fedhly ${ }^{1, *}$ and Waguih ElMaraghy ${ }^{1}$ \\ 1 Intelligent Manufacturing Systems Centre (IMSC), University of Windsor, \\ 1212 Centre for Engineering Innovation (CEI 1212) \\ 401 Sunset Av., Windsor, Ontario, N9B 3P4, Canada \\ * Corresponding author: fedhly@gmail.com
}

\begin{abstract}
Dealing with complex systems often involves a layering (e.g.: hierarchical) representation that is prevalent throughout engineering. This layering appears in various disciplines in a variety of forms. Concurrent design refers to a generalized methodology for mapping from the functional requirements and conceptual model to blueprints in various domains. Cyber-Physical, hence complex, system include mechanical, electrical, sensors, computer, data, user interface, and external factors. The system is capable of acquiring historical data, receiving real-time sensory status, adapt accordingly while interacting with the environment. The purpose of this paper is to introduce a novel design methodology approach for smart complex systems such as cyber-physical products and machines. The potential impact of this methodology is to provide a common design approach for any smart system. It can also be used to calculate system complexity in the context of the coupling index. Additionally, it can reduce the compatibility issues at the early system design level.
\end{abstract}

Key words: Product Design, Cyber-Physical Product Design, Design Methodology.

\section{1- Introduction}

With the developing of new generation customer requirements and technology advancement, rapidly changed product design is becoming a necessity. Accordingly, many challenges have been evolved to product design in the context of smart manufacturing systems development towards the next generation industry. Smart technology era requires a new design methodology. The smart sensory systems from machines, people, and material is being interconnected in an integrated environment. Yet, there is no machine able to make decision in a way human can, especially in synthesis level [1, 2]. Instead, the new generation smart machines, computer microprocessor driven devices and artificial intelligence integration, can be used to aid decision making through data acquisition and processing [3].

The purpose of this research is to explore design methodologies in order to identify research gaps, then to propose a design methodology to serve as a framework that helps in multi-discipline product design with relatively new application of semantic block design approach which can enable a unified system representation according to the viable connectivity where applicable at conceptual design phase development. This tool can benefit multiple parties at different product design domain. For example, it can help link machinery manufacturer to all stake holders including software developer, mechanical designer, system 
designer, control designer, environmental and compliance designer in order to concurrently collaborate during product development. Product conceptual design phase represents the foundation of all future functionality and process. The information and documentations at this step can be a crucial for further product design and development.

\section{2- Literature Review}

A selected relevant literature review will be carried out in this section. It will be focused on the methodology which is used to back up the invented methodology.

\section{A. Design and Process Progress}

Design process, usually, starts with conceptual scheme which is the basic step for any invention where all further phases are greatly depending on this step. It shapes a conceptual functionality for the innovative solution toward specific customer requirement. Typically, it contains basic details of the design phase. The main components in this design phase is the model of physical action, constraints and limitations. Designer outline a descriptive concept according to functional requirements where it answers what the product should do but not how. It is agreed that design is one of the core engineering roles [4].

According to Zang's, et al. [4] survey in cyber-physical system design methods, the system integration during conceptual phase is not yet achievable. It is believed that it is related to continuous trend of using the same methodology, or available tools, of the last century where physical functions were the core of the product. i.e. TRIZ method have been developed in 1946 while Suh had documented axiomatic design as method in 1980 [5]. However, new product paradigm exceeds electro mechanical aspects to computational, data, and external factors like human and environmental performance.

\section{B. System Behavioural Challenges}

It is agreed with Tomiyama, et al. [6] that behavioural capabilities are very important aspects of smart product features. Although resilience is a crucial behaviour of these systems, it is believed that system stability represents the ultimate design goal, robust design. however, some practices are used to improve such behavioural vulnerability like fault tolerance. Furthermore, smart complex systems can be approached as multidisciplinary network system to improve its stability carried out by Bahreini, et al., [7]. They insert a random delay between the controller, sensor and actuator. The method can be generalized and used to validate design stability at block level where timeline of the response is tightly tolerated or an external factor had not been considered during design phase. It is noticed that behavioural challenges are drawing an attention at empirical research, but not at system design level. It might be related to the multi-disciplinary systems nature where the challenges emerge after developing a conceptual model.

\section{Multi-Disciplinary Product Design}

In this paper, a multi-disciplinary product may refer to any cyber-physical machine or device (product) that consists of mechanical, electrical, sensor, and data. External components can also be a part of the system to represent user, environment, and the affected stockholders or components. It is often referred to as smart system, smart device, industry 4.0 machine, or intelligent device/system. As device is a group of connected components, the term system may 
also refer to a wider definition which includes, but it is not necessarily limited to, devices.

Multi-disciplinary device is usually a complex system, where different type of engineering design may be required, in different domains, according to the design requirements. Besides possibly being multi-disciplinary, a device may be designed by a company that will do only conceptual and embodiment models, but the detailed design may be completed using different physical principles (e.g. mechanical, electrical, pneumatics, etc.). The assembly of the various components into may get done by a third parties. As a result, a system engineering, and simultaneous or concurrent engineering approach is used as a design strategy in order to manage such multi-party complex operations. It is usually an iterative procedure where design gets improved by mitigating certain drawbacks or limitations of current model or design. An important aspect of concurrent design is that it can be developed simultaneously despite the fact of its multiple components are dependent on each other. This practice raises the potential great source of compatibility as a result [8].

Casner, et al. [9] proposed a mechatronic design approach especially important in the embodiment phase based on Verein Deutscher Ingenieure, VDI 2206 [10] model. VDI 2206 model is an interconnected $\mathrm{V}$ shape operations where design levels are connected in circular, iterated, a long progress levels as it shown in Fig.1. It is agreed with Zheng, et al. [11] this model does not evidently seem to support multiple engineer/designer/developer even though it addresses multi-discipline "design domains".

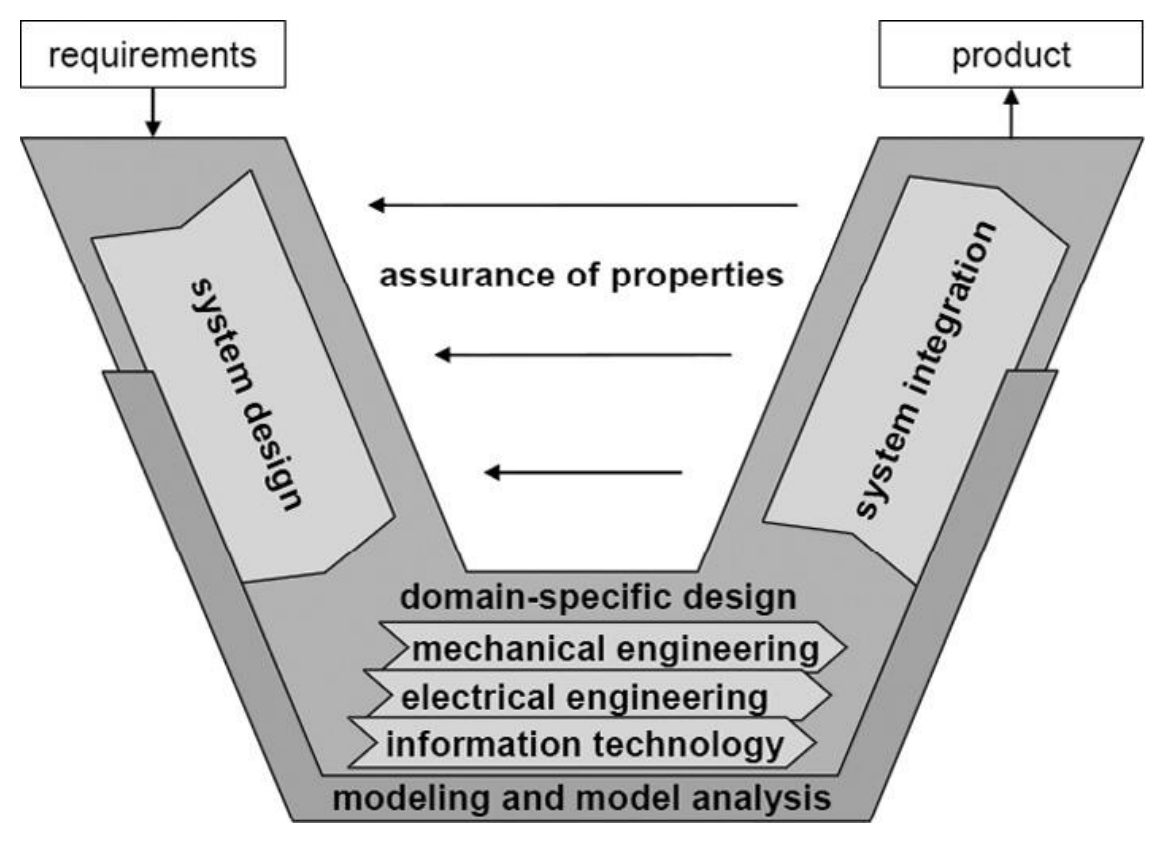

Fig.1 design methodology recommended in [10]

It is however partially agreed with Casner, et al. [9] that their proposed methodology is built on the grounds of VDI 2206 [10] but the resulted methodology is more novel. By comparing both approaches, it can be concluded that VDI2206 [10] methodology is more iteration based, macro-cycles, than specifying design phases or even systems functionality, while Casner, et al. [9] approach, shown in Fig.2, is more about design process details where functionality is the core of each level with associated optimization step within the integration phase. The proposed methodology of Casner, et al. [9, p. 35] seems to offer more than design 
optimization during the embodiment phase. It is believed that it also provides an outline before the embodiment phase within Step 1.2: "Define Global Functions of the Overall System". Furthermore, it can be considered as a design framework where the integration takes place by validating the outcome design in Phase\#3 to the earlier design Phase\#1 as optimization objectives can be met. The main drawback of this methodology is that it does not specify integration process, or even optimization, during building the product or prototyping stage. Also, data technology has not been validated in the case study example. Furthermore, it is mainly a functional based approach.

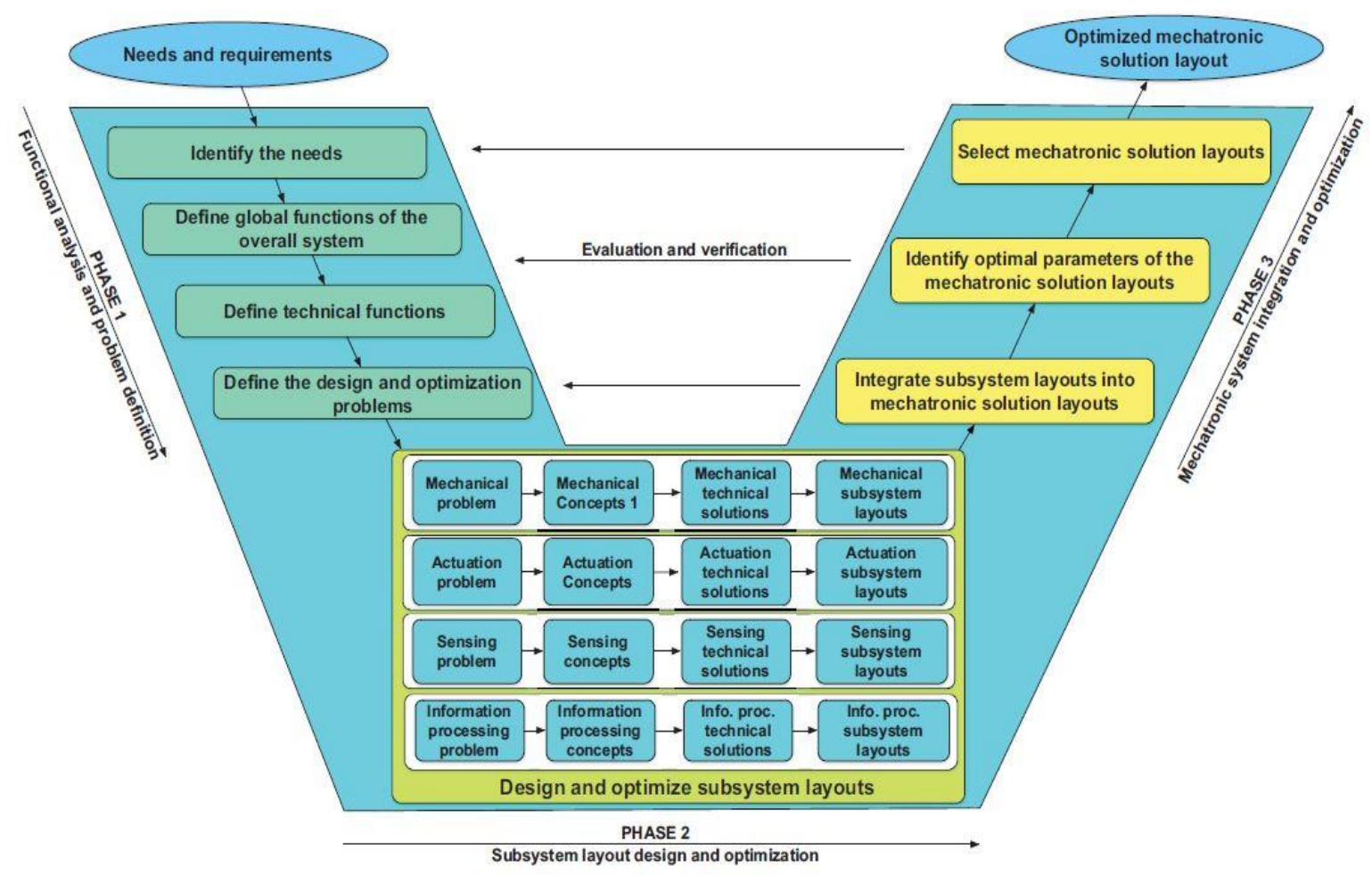

Fig.2 Design optimization integrated approach in [9]

Consequently, it is strongly believed that Casner, et al. [9] methodology is a well advanced design methodology and overcome the main drawback of identifying the multiple design domain, specifically addressing four functional domains, where other approaches are mainly intended to serve the mechanical design domain, e.g.: US Department of Transportation[12]. It is illustrated in Fig.3 and is not equivalent, albeit the fact that is shows apparent similarities of shape and integration. 


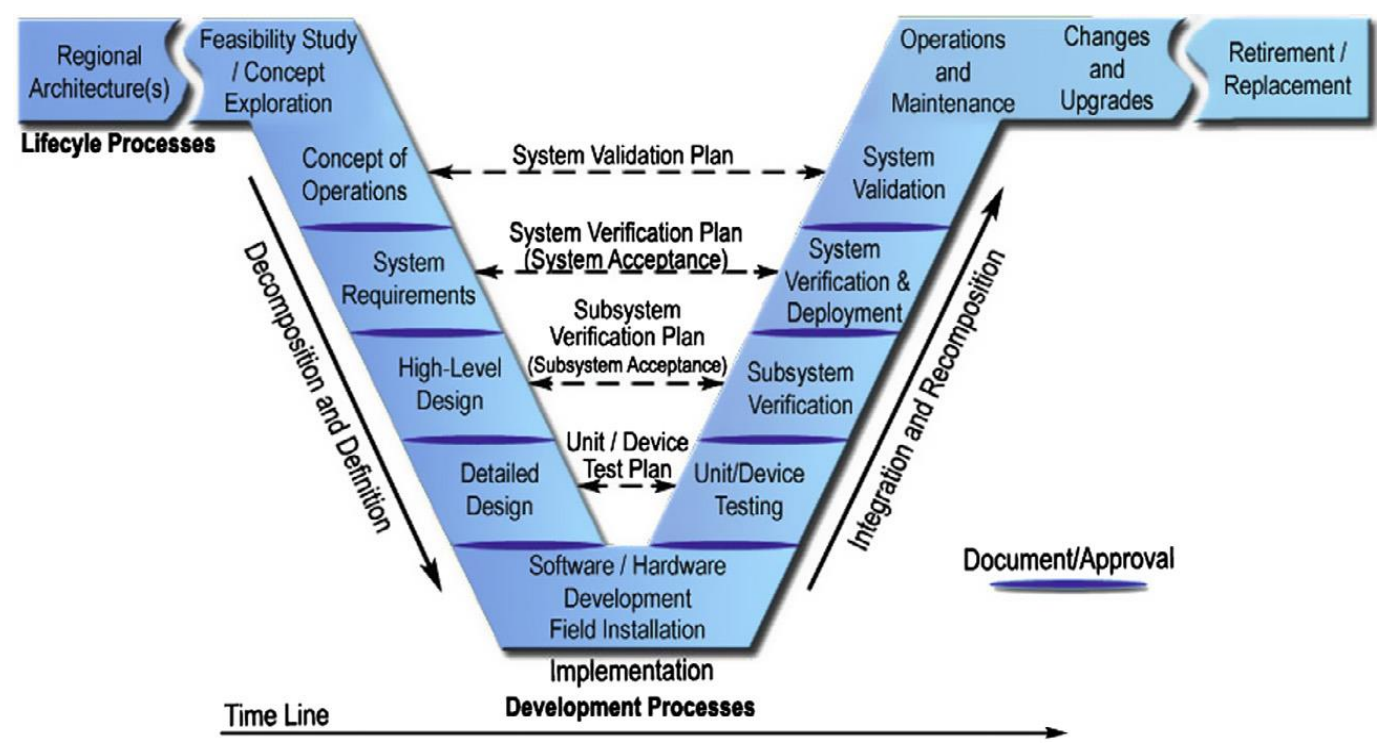

Fig.3 concurrent V model used by US Department of Transportation [12, p. 11]

\section{Literature Conclusion and Discussion}

1) System Integrity: The main objective of machines is to ease human life by performing tasks that are not easy for humans, as related to physical, economical, psychological, and geometrical aspects. Cyber-Physical systems are the state of art products that utilize the latest technology to achieve a standalone systems' performance as an ultimate goal. However, there are some major foreseen challenges that make such systems vulnerable and risky. For example, electric power and data are crucial components for cyber-physical systems to perform its designated tasks. Also, as a device, needs power to operate sensor and actuators. On the other hand, it needs effective data technology to optimally complete the required function. These challenges can be more complicated if cloud computing and big data are interrupted for any reason. Cyber-security is another system integrity challenge which can present the additional complexities of smart systems.

2) Affordance: Human-Machine interaction is still at master slave mode. Yet, human cannot afford to live with machines for many reasons. Socially, for human factors and safety consideration proximity to a machine may still be a source of injury due to an unexpected system failure. Nevertheless, machine mistakes are still not tolerable despite the fact machines may statistically perform more efficiently. This aspect might be related to social aspects but it may also be related to job security and fears by the human who are affected by smart machines. Design failures can be very costly in terms of economic, social, and feasibility if not carefully considers all stakeholders.

\section{E. Social Acceptance}

Another challenge can lay in social aspect of implementing smart systems. In addition to job security threats, smart systems need heavy infrastructure investments so far. This investment is beyond any single company budget and interest at the same time. The current 
debates are in favor of governmental investments which makes implementation more difficult challenge. Budget, tax, jobs are enough challenges for any government to solve than any extra spending in companies' favor infrastructure technology. It I highly addressed challenge which requires extra investigation. The whole future of smart systems relays on the outcome of such investigations.

\section{F. Design Challenges of smart system}

The main essential challenge of cyber-physical systems are the principal requirements of the system itself aside from the functional requirements. Principal requirements can be crucial even before conceptual design. Principal requirements represent prerequisite condition in order to implement system design. Principal requirements can be distinguished from basic requirements, which represent system readiness like connectivity and data technology. The principal requirements can be defined as: system's functional limitation, which include: boundaries, default state, fail safe mode, environmental restrictions, required spatial limitations, ... and so on. It is believed that clearly defined principal requirements result in robust system behavior. Also, it can provide a pre-defined fail-safe state for each system component in order to increase system reliability. A schematic diagram of customer domain, functional domain and system domain is shown in Fig.4.

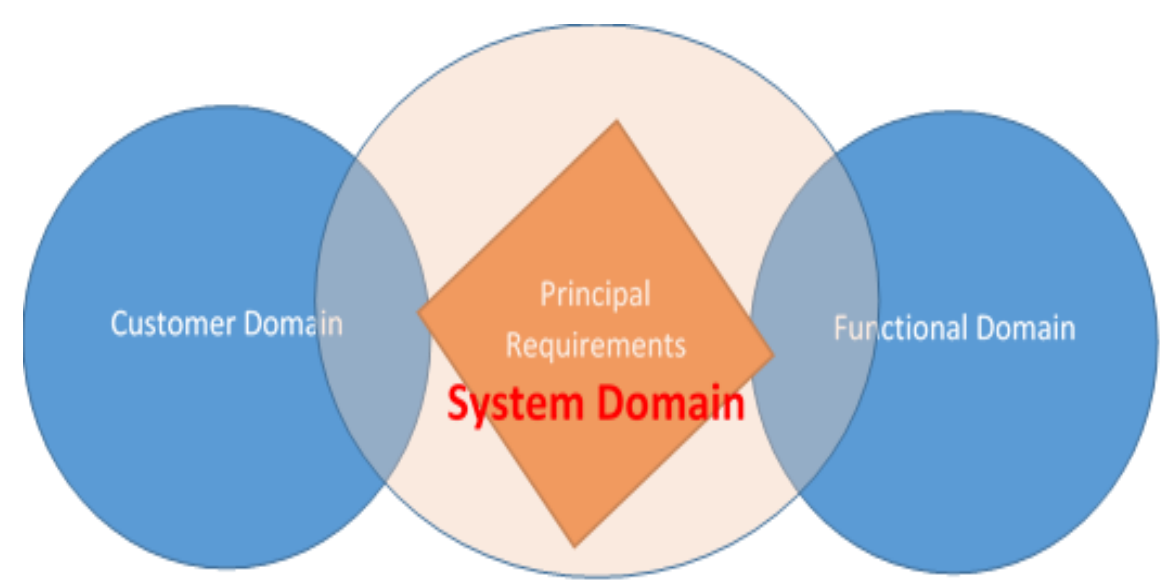

Fig.4 Cyber -Physical Product Design Principal Requirements

\section{3- Proposed Design Methodology}

According to the conducted literature review, cyber-physical systems design is the ongoing project toward the next generation products. So far, the system consists of concept that solves the basic need which is realized by a mechanic component with a kinematics controlled by electrical components receiving instructions from a computer that acquires feedback data about the mechanical device from attached sensors supported by historical data in order to optimize performance by using adaptation techniques. This system is interacting with the external factors and environment. The product also affects the interconnected components as a result of its functionality. Fig. 5 shows a projected diagram of the cyber physical device components. 


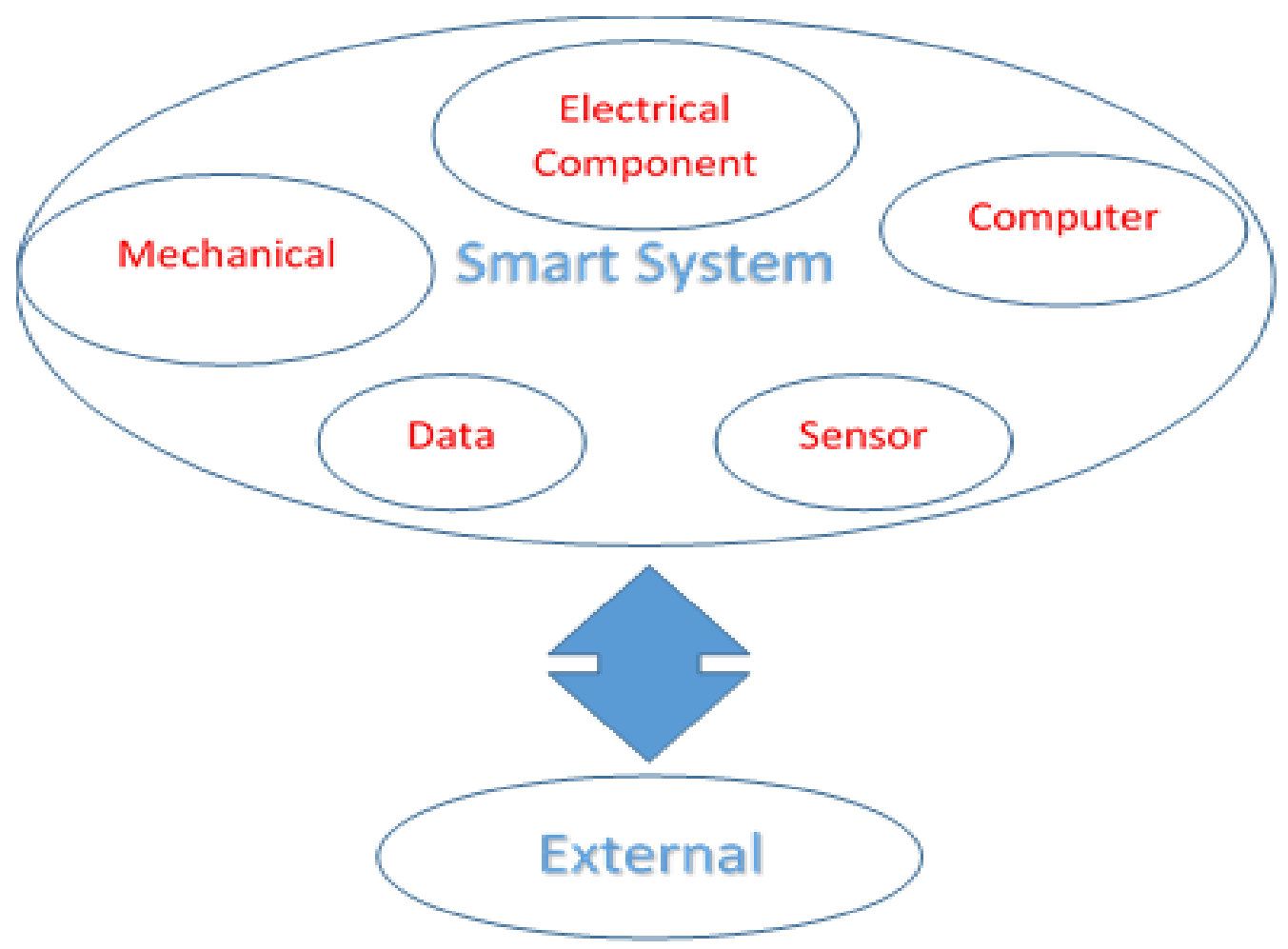

Fig. 5 Cyber-Physical system components

Such system motivated a V shape design methodology which has been developed based on VDI 2206 [10], US Department of Transportation [12] and Casner, et al. [9]. The invented $\mathrm{V}$ shape design methodology combine specification, prototyping and optimization advantage with the consideration of concurrent multiple party engagement during design and development phases. Fig. 6 shows the proposed design methodology. The main contribution in design methodology steps is highlighted in red colour. Components Conceptual Design and Abstractional design, represent unified design tools to be used among multiple disciplinary designers as information sharing platform within the extended conceptual level of details, and its corresponding validation stages in the integration stage. The multidisciplinary concepts come right after deciding the main conceptual design. It is necessary for each design component to collaborate with all others in order to produce successful designed product as a result. A case study will be carried out to validate the proposed methodology. 


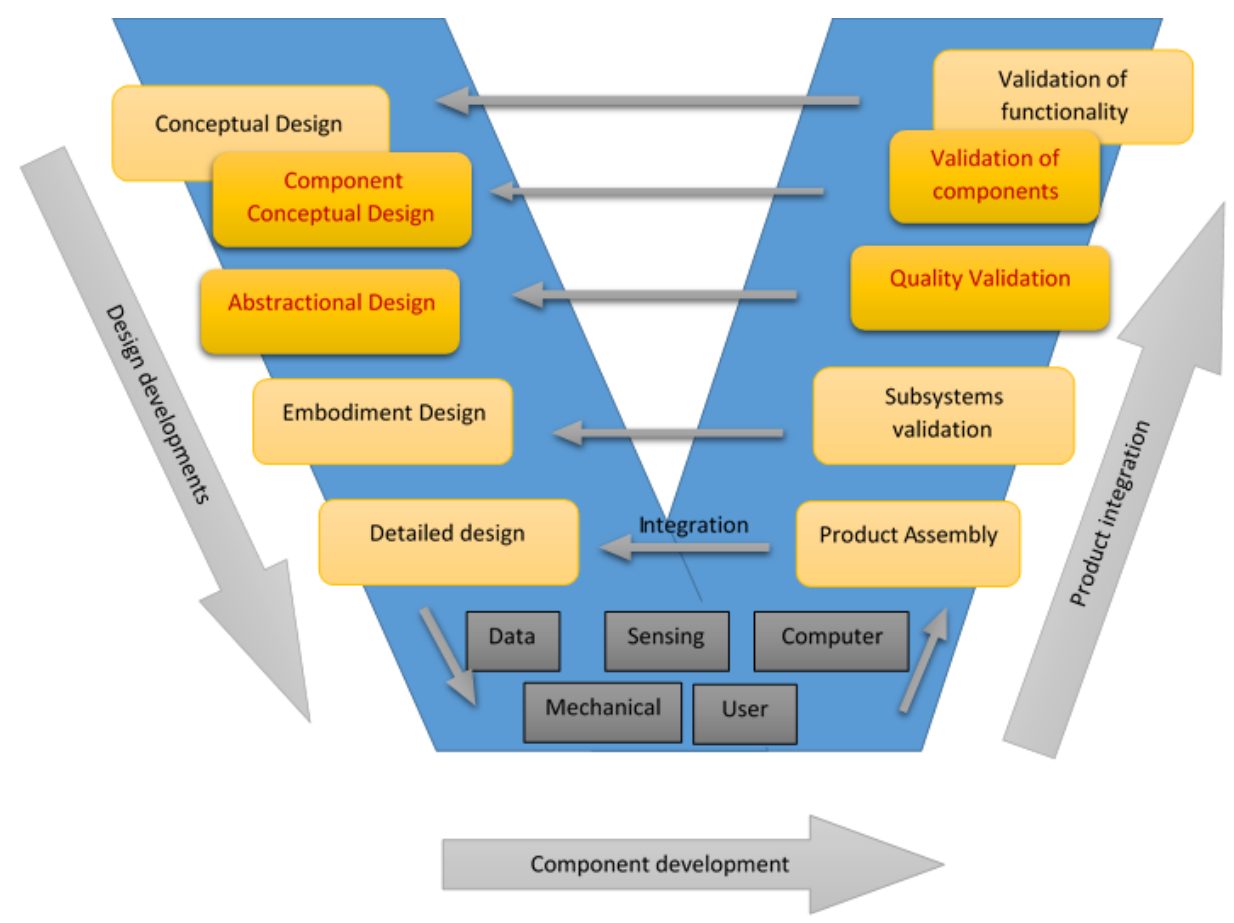

Fig.6 Concurrent Multidiscipline product design and evolution methodology

The proposed methodology suggests that components can be developed separately, by different designers and possibly several be builders. It starts from conceptual level through embodiment level to the detailed design level as shown in Fig.7.

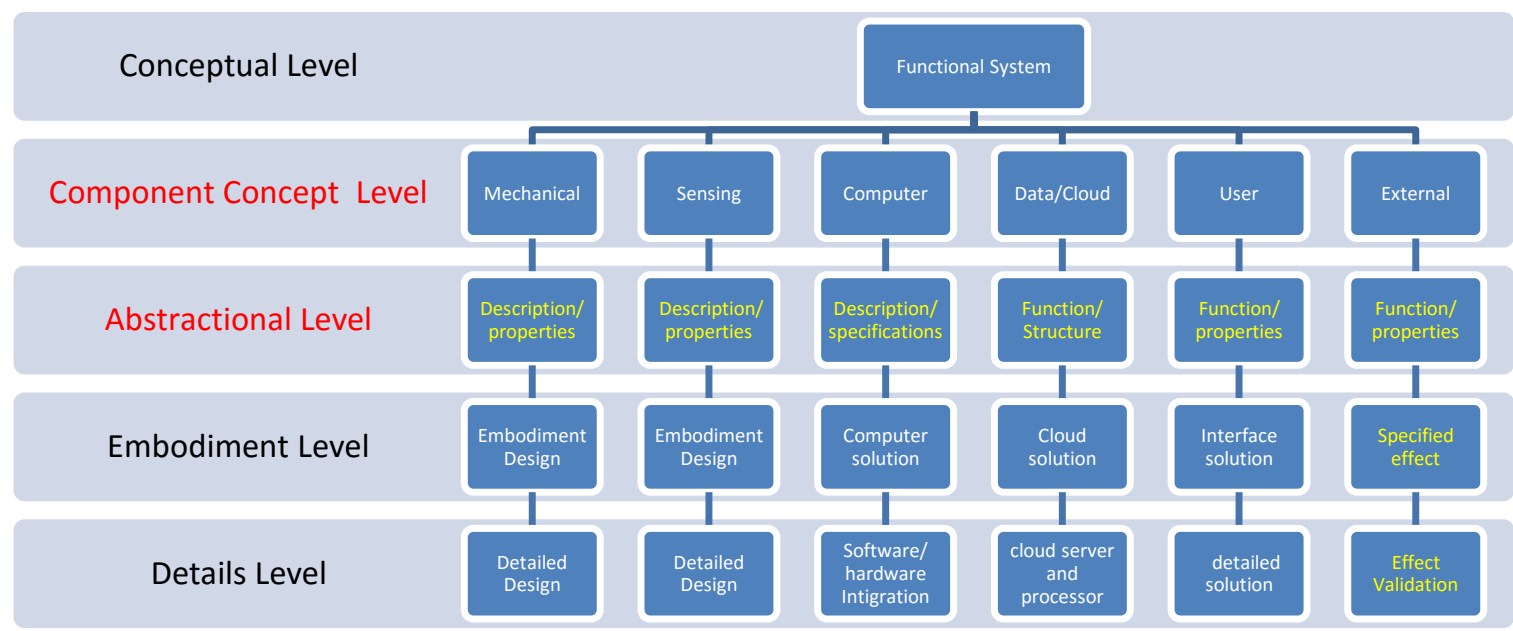

Fig.7 Product's components to be used by multiple design development party

\section{4- Case Study: Car Windshield Defroster Design}

For the purpose of explanation and validation of the proposed methodology framework, a conceptual design of a car windshield will be discussed in this section. Functional requirements are presented in a block diagram shown in Fig.8. 


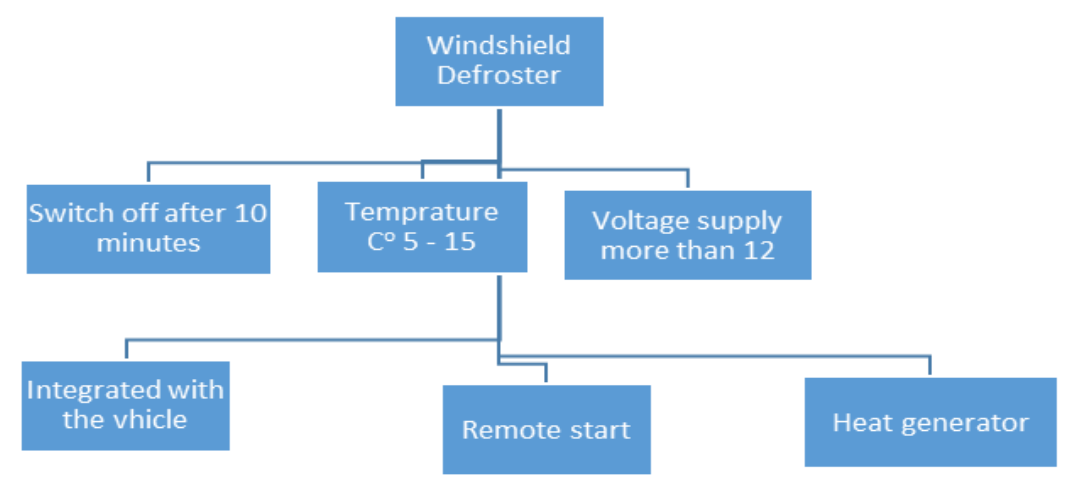

Fig.8 Windshield functional requirements diagram

A conceptual design has been developed by using Morphographic matrix. It is used to synthesize a modular conceptual design to satisfy the functional needs in Fig. 8. Three design alternatives have resulted: A, B and C. Table 1 illustrates the suggested concepts and relevant combinations.

Table 1 Morphographic table for windshield design alternatives

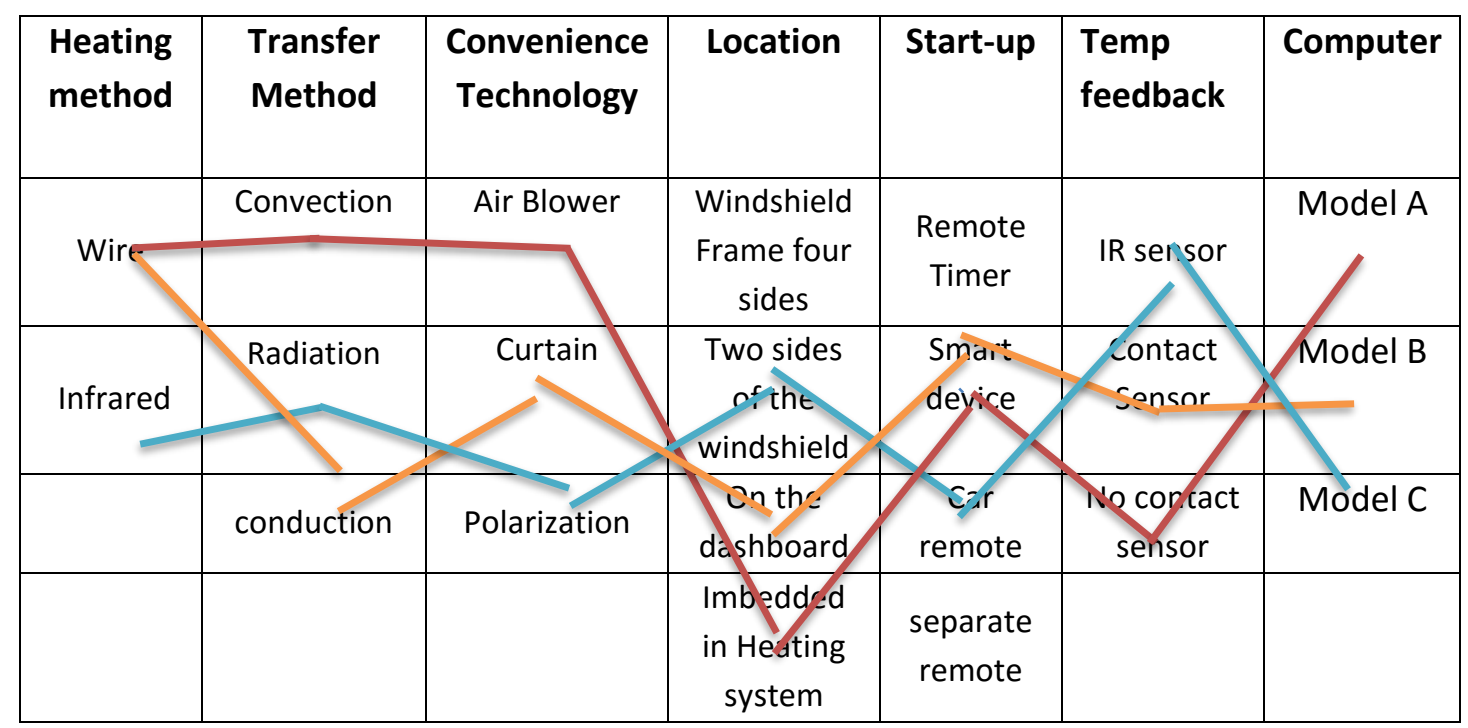

Product A

Product B

Product C

A component conceptual block model is developed after the main conceptual model, where every component may have its own conceptual model as shown in Fig.9. 


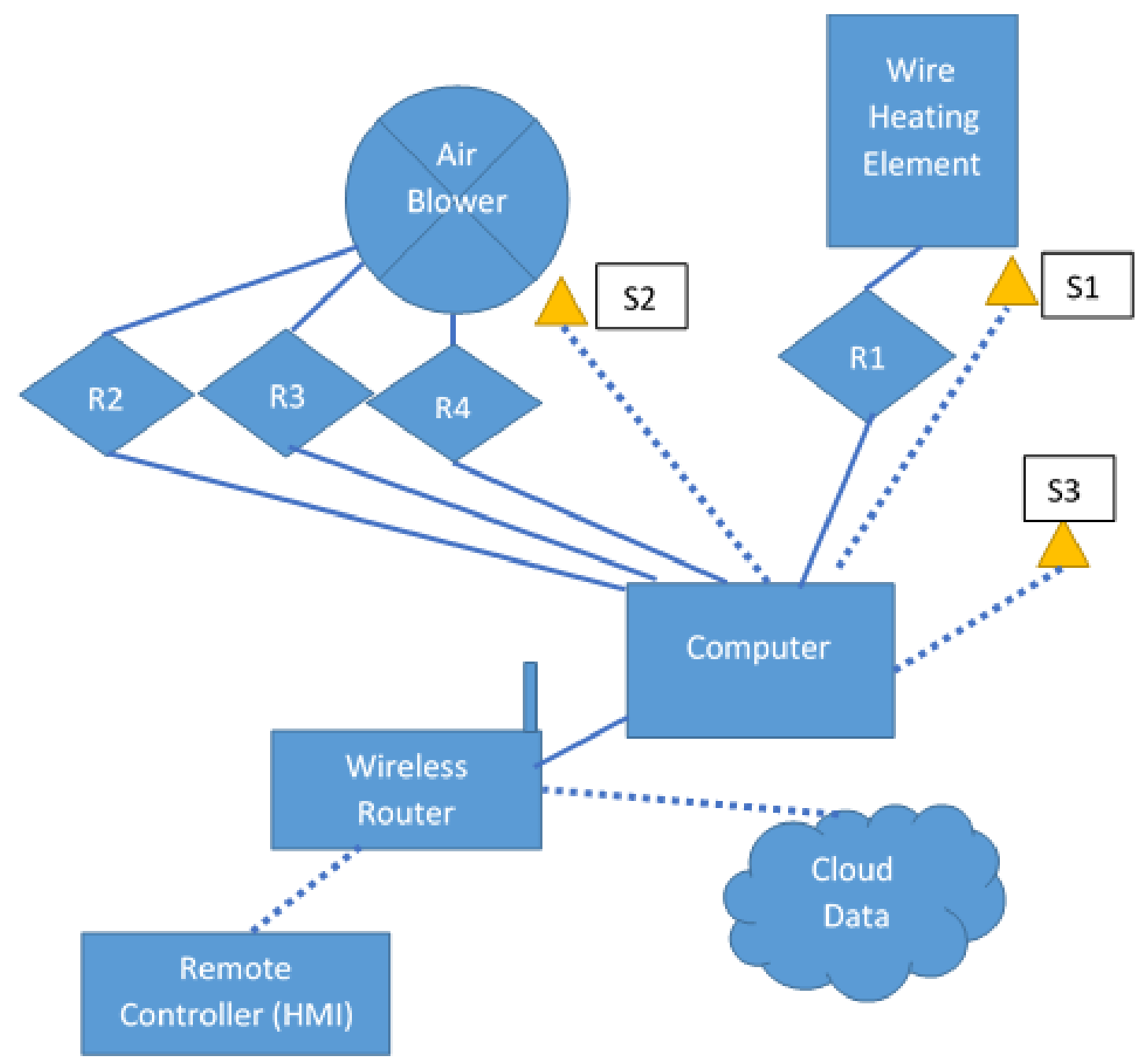

Fig.9 Component Conceptual Block Design for the proposed car windshield

Consequently, the main drawback of the design in Fig. 9 is that it cannot determine the multi-disciplinary design process. Instead, it can illustrate the main components and the potential conceptual linkage between components. Accordingly, a semantic block design has been developed to illustrate the components described in Fig. 7 and 9. The categorization shown in Fig.10 can be used to facilitate the multiple design party approach. An important component of the product design is the external components. It can be designed by environmental engineer where stack holders of the product can be considered or even by multiple engineer like safety and geological engineers. External components may include the affected component, by the output of the function, like the windshield in this case study. Codes, rules and regulations can also be used as external factors to be shared among all designers in order to comply with them. 


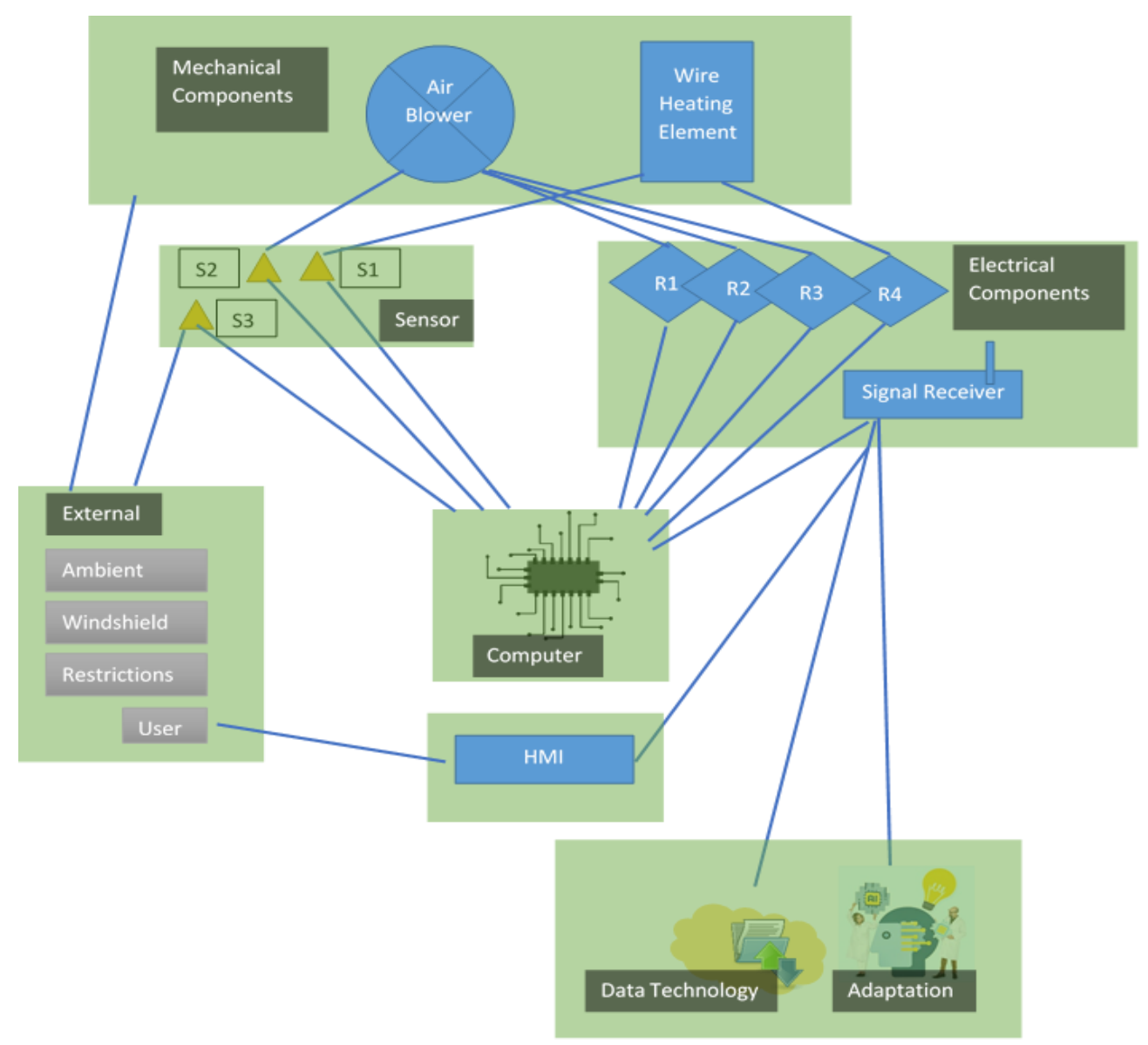

Fig.10 Semantic block design for the proposed car windshield defroster

\section{5- Conclusions and Future Work}

\section{A. Conclusions}

Technology advancement is driving more complex multi-disciplinary product innovation. A proposed systematic multiple party design methodology framework can be used as a platform to develop products concurrently and also used to validate and integrate product within its corresponding design phase. Such systematic methodology can benefit by reducing design iteration by providing a common road map work-flow, i.e. a methodology framework, to be shared among multi designers. It can also improve technical communication among parties. However, although the proposed methodology is validated by a case study, it is considered in developing stages and needs more developments to satisfy the technical multidisciplinary domain technology advances.

\section{B. Future work}

The proposed methodology covers product development from functional requirements to the manufacturing stage. The future plan is to extend the methodology to include the complete products lifecycle from customer-needs to grave. It can be promising to be used as a base to develop design software. 


\section{References}

[1] G. Schuh, R. Anderl, J. Gausemeier, M. ten Hompel and W. Wahlster, "Industrie 4.0 Maturity Index. Managing the Digital Transformation of Companies (acatech STUDY)," Herbert Utz Verlag, Munich, (Eds.) 2017.

[2] F. Tao and M. Zhang, "Digital Twin Shop-Floor: A New Shop-Floor Paradigm Towards Smart Manufacturing," IEEE Access, vol. 5, no. 20, pp. 418-427, 2017.

[3] F. Tao, F. Sui, A. Liu, Q. Qi, M. Zhang, B. Song, Z. Guo, S. C.-Y. Lu and A. Y. Nee, "Digital twin-driven product design framework," International Journal of Production Research, vol. 57, no. 12, pp. 3935-3953, 2019.

[4] C. Zang, J. L. Duigou, P. Hehenberger, M. Bricogne and B. Eynard, "Multidisciplinary Integration During Conceptual Design Process: A Survey on Design Methods of Cyber-Physical System," In DS 84: Proceedings of the DESIGN 2016 14th International Design Conference, pp. 1625-1634, 2016.

[5] E. M. Benavides, Advanced Engineering Design - An Integrated Approach, 1 st ed., Elsevier, 24 November, 2011.

[6] T. Tomiyama, E. Lutters, R. Stark and M. Abramovici, "Development capabilities for smart products," CIRP Annals Manufacturing Technology, vol. 68, no. 2, pp. 727-750, 2019.

[7] M. Bahreini, J. Zarei, R. Razavi-Far and S. Mehrdad, "Robust finite-time stochastic stabilization and faulttolerant control for uncertain networked control systems considering random delays and probabilistic actuator faults.," Transactions of the Institute of Measurement and Control, vol. 41 , no. (2), 3550-3561, 2019.

[8] D. Bradley, "Mechatronics - More questions than answers," Mechatronics, vol. 20, no. 8, pp.827-841, 2010.

[9] D. Casner , H. Remy , J. Renaud and D. Knittel , “An Optimization-Based Embodiment Design Approach for Mechatronic Product Development," The Open Automation and Control System Jurnal, vol. 9, no. Suppl-1, M4, pp. 27-47, 2017.

[10] VDI 2206 , "Verein Deutscher Ingenieure - Entwicklungsmethodik für mechatronische Systeme (design methodology for mechatronic systems)", 2004.

[11] C. Zheng, M. Bricogne, J. Le Duigou and B. Eynard, "Survey on mechatronic engineering: A focus on design methods and product models," Advanced Engineering Informatics, vol. 28, no. 3, pp. 241-257, 2014.

[12] Department of Transportation, Office of Operations, "Systems Engineering for Intelligent Transportation Systems," January 2007. [Online]. Available: <https://ops.fhwa.dot.gov>. [Accessed 23 April 2020].

[13] M. V. \&. M. V. Sibalija T, “An intelligent approach to robust multi-response process design,” International Journal of Production Research, vol. 49, no. 17, p. 5079-5097, 2011. 